\title{
ESTUDIO DE MERCADO PARA LA CONSTRUCCIÓN DE UN RESORT TURÍSTICO.
}

\section{MARKET STUDY FOR THE CONSTRUCTION OF A TOURIST RESORT.}

Gilberto Blanco González, MBA.

Máster en Administración de Empresas (Cuba).

Docente Titular en la Escuela Superior Politécnica Agropecuaria de Manabí (ESPAM),

Ecuador. Coordinador de la Unidad de Docencia, Investigación y Vinculación del Hotel Higuerón de la ESPAM. gblanco@espam.edu.ec

Mabel Font Aranda, PhD.

Doctora en Ciencias Geográficas (Cuba).

Docente de la Escuela Superior Politécnica Agropecuaria de Manabí (ESPAM), Ecuador. mabelfontaranda@gmail.com

Juan César Villacreces Viteri, MSc. Máster en Gestión Turística (Cuba).

Director de la carrera de Turismo en la Escuela Superior Politécnica Agropecuaria de Manabí (ESPAM), Ecuador. jvillacreces@espam.edu.ec

\section{ARTÍCULO DE INVESTIGACIÓN}

Recibido: 26 de diciembre de 2016.

Aceptado: 27 de febrero de 2017.

\section{RESUMEN}

La presente investigación tiene como objetivo general desarrollar un estudio de mercado para la construcción de un resort que fomente el desarrollo turístico rural del cantón Chone. La metodología utilizada se fundamentó en el análisis del criterio de cuatro autores, Vela (2012), Espinoza (2013), Fassler (2013), Salazar y Yánez (2014); realizando como primera fase un diagnostico situacional de las generalidades, antecedentes, atractivos y características de los alojamiento turísticos y a su vez recopilando información 
de las diferentes actividades que se desarrollan en dicha región, desde la actividad agrícola y sus principales productos; la actividad ganadera, y el comercio que se ubica en el tercer renglón económico del territorio manabita. La segunda fase consiste en el análisis de la oferta, demanda y competencia en el que se determinó las preferencias del turista, se evaluó los diferentes alojamientos turísticos del cantón y de las regiones próximas al mismo, a su vez se identificaron los gustos y exigencias de los visitantes, obteniéndose como resultado que la demanda se direcciona a clientes nacionales; como tercera y última fase se diseñó el mix del marketing del producto en el que se planteó estrategias de promoción, publicidad, el posicionamiento del logo y del slogan, además del precio y los diferentes canales de distribución y promoción para sustentar el presente trabajo. Sobre la base de los resultados obtenidos en la encuesta, se pudo constatar que hay una aceptación del $83 \%$ de turistas los que estiman que es factible la creación de un Resort en el cantón Chone.

Palabras clave: estudio de mercado, metodología, demanda, competencia, oferta, mix del marketing.

\section{ABSTRACT}

The present research has as general objective to develop a market study for the construction of a resort that foments the rural tourism development of the canton Chone. The methodology used was based on the analysis of the criteria of four authors, Vela (2012), Espinoza (2013), Fassler (2013), Salazar and Yánez (2014); Carrying out as a first phase a situational diagnosis of the generalities, antecedents, attractions and characteristics of the tourist accommodation, in turn gathering information of the different activities that are developed in that region, from the agricultural activity and its main products; The livestock activity, and the trade that is located in the third economic area of the Manabí territory. The second phase consists of the analysis of the supply, demand and competition in which the preferences of the tourist were determined, the different tourist accommodations of the canton and of the regions close to it were evaluated, in turn the tastes and requirements of the visitors, obtaining as a result that the demand is directed to national clients; As a third and final phase, the product marketing mix was designed in which promotion and publicity strategies were proposed, the positioning of the logo and the slogan, as well as the price and different distribution and promotion channels to support the present work. Based on the results obtained in the survey it was found that 
there is an acceptance of $83 \%$ of tourists who believe that it is feasible to create a Resort in the Chone cantón.

Keywords: Market study, methodology, demand, competence, offert, marketing mix.

\section{INTRODUCCIÓN}

Hoy por hoy, en el Ecuador se ha catalogado al eje turístico como una oportunidad importante de dinamización para la economía del país, en el Plan Nacional del Buen Vivir 2013-2017, establece que el turismo es uno de los sectores prioritarios para la atracción de inversión nacional y extranjera, siempre y cuando no desplace las iniciativas locales y en concordancia con la Constitución de la República se establezcan propuestas que reconozcan los derechos de la naturaleza y las bases para un desarrollo sostenible (MINTUR 2015).

Como contraste de lo anteriormente planteado en el Ecuador existe déficit en la oferta de hospedaje; por esta razón en determinadas ocasiones como feriados, fines de semanas y fines de años, no hay capacidad para la demanda, lo cual genera ofertas débiles y poco apetecibles para el mercado; por tal motivo es necesario buscar el desarrollo de nuevos productos, la creación de instalaciones de ocio, la formación y la cualificación de los servicios, desarrollar líneas de investigación y análisis que contemplen las características de la oferta, la demanda y que contribuyan a satisfacer las necesidades y exigencias de los turistas.

En el documento de planificación PLANDETUR 2020, el Ecuador dispone de un potencial turístico inigualable a nivel mundial, por su diversidad, su naturaleza y sus culturas, las que ofrecen oportunidades que han sido muy poco aprovechadas y que están siendo explotadas por empresas, como por ejemplo la creación de centros de hospedaje comunitarios y Resort en los cuales se brinden servicios diferenciados. A tal efecto es necesario tener presente que el potencial turístico resulta afectado cuando se desarrollan proyectos gigantescos y que no se enfocan en el criterio del posible visitante, y que no resultan atractivos para el tipo de demanda existente.

La Provincia de Manabí cuenta con infraestructura hotelera, pero por el desconocimiento de las necesidades y exigencias del turista, existen lugares que no están completamente estructurados para brindar un servicio de calidad.

El alojamiento es el componente principal para el desarrollo del turismo y en la provincia de Manabí se puede encontrar una basta gama de ofertas hoteleras con incomparables características y con servicios dirigidos a diferentes targets. 
En el cantón Chone existe una limitada infraestructura turística, siendo insuficiente para asumir los viajeros que se trasladan a este cantón, además el nivel de calidad de las instalaciones dista de cualquier tipología del alojamiento para que el turista se sienta confortable y por tanto ello puede limitar el movimiento turístico en el cantón; cuestión que se tuvo en cuenta al evaluarse la categorización y clasificación registrada en el catastro nacional de Ecuador 2016 en el que se refleja que los alojamientos turísticos existentes en el territorio oscilan entre segunda y tercera categoría y solo en la tipología de hostales. En base a estas apreciaciones es que surge la idea de contribuir al incremento del alojamiento es a través de la creación de un Resort que fomente el desarrollo turístico rural en el cantón Chone.

El propósito de elaborar un estudio de mercado para la construcción de un resort que fomente el desarrollo turístico rural en el cantón Chone, se justifica ya que la propuesta busca promover e impulsar el turismo enfocándose en las nuevas alternativas turísticas sostenibles, sugiriendo actividades hoteleras diferenciadas, de diversificación y por ende el involucramiento de los habitantes circundantes al área de estudio, contribuyendo en la dinamización socioeconómica, medioambiental de los recursos naturales de una manera sustentable con el respaldo de las organizaciones locales como la Junta parroquial, y el GAD del cantón Chone.

\section{MATERIALES Y MÉTODOS}

La investigación tuvo como alcance la provincia de Manabí, en la cual se tomaron en cuenta tres cantones para la realización del trabajo. De acuerdo a información de la página del gobierno provincial de Manabí, la provincia se encuentra ubicada en el noroeste del Ecuador.

Para el desarrollo metodológico de la investigación se tomó como referencia a trabajos que posean alguna similitud a lo propuesto en esta investigación, es decir los que tienen como resultados la realización de un estudio de mercado, es así que se citan los trabajos como la creación de un nuevo producto, que sirven como punto de partida para crear una matriz conceptual que sustente el diseño metodológico propuesto. La metodología proyectada ayudara a que la información consultada sea verídica, lo cual contribuirá con el desarrollo de la propuesta planteada. A continuación se describe el cuadro de análisis de la metodología de varios autores como: Vela (2012), Espinoza (2013), Fassler (2013), Salazar y Yánez (2014), quienes desarrollan metodos aplicables a esta investigación. 


\section{Revista Científica ECOCIENCIA}

Tabla 1. Análisis de la metodología.

\begin{tabular}{|c|c|c|c|}
\hline Vela (2012) & Espinoza (2013) & Fassler (2013) & $\begin{array}{c}\text { Salazar y Yánez } \\
(2014)\end{array}$ \\
\hline $\begin{array}{l}\text { Evaluación del } \\
\text { potencial }\end{array}$ & 1. Antecedentes & $\begin{array}{l}\text { 1. Caracterización } \\
\text { del lugar }\end{array}$ & $\begin{array}{l}\text { 1. Diagnóstico del } \\
\text { entorno }\end{array}$ \\
\hline $\begin{array}{ll}\text { 2. } & \begin{array}{l}\text { Estudio de } \\
\text { mercado }\end{array} \\
\end{array}$ & $\begin{array}{ll}2 . & \text { Diseño de la } \\
\text { investigación }\end{array}$ & $\begin{array}{ll}\text { 2. } & \begin{array}{l}\text { Estudio de } \\
\text { mercado }\end{array} \\
\end{array}$ & $\begin{array}{l}\text { 2. Investigación de } \\
\text { mercado }\end{array}$ \\
\hline $\begin{array}{l}\text { 3. Diseño técnico del } \\
\text { producto }\end{array}$ & 3. Estudio de mercado & $\begin{array}{l}\text { 3iseño de } \\
\text { paquetes } \\
\text { turísticos }\end{array}$ & $\begin{array}{l}\text { 3. Análisis de oferta, } \\
\text { demanda y } \\
\text { competencia }\end{array}$ \\
\hline $\begin{array}{ll}4 . & \text { Estudio } \\
& \text { administrativo }\end{array}$ & 4. $\quad$ Mix de marketing & $\begin{array}{l}\text { Estrategias de } \\
\text { comercialización }\end{array}$ & $\begin{array}{l}\text { 4. Estrategias de } \\
\text { desarrollo }\end{array}$ \\
\hline $\begin{array}{ll}\text { 5. } & \text { Estudio } \\
& \text { económico } \\
\end{array}$ & 5. Estudio ambiental & $\begin{array}{l}\text { Factibilidad } \\
\text { económica }\end{array}$ & $\begin{array}{l}\text { Costo total de la } \\
\text { propuesta }\end{array}$ \\
\hline & & & $\begin{array}{l}\text { Análisis de } \\
\text { impactos }\end{array}$ \\
\hline
\end{tabular}

Fuente: elaboración propia.

Después de plasmar las diferentes metodologías propuestas por los autores antes mencionados, cabe realizar la propuesta a partir del desarrollo de una matriz conceptual cuyo objetivo es analizar cada una de ellas y comparar los criterios similares y aquellos que los diferencian y de esta forma proponer una propia , cuyo objetivo es analizar los métodos de cada uno de los autores citados en el cuadro 2, además de plasmar una definición y comparar los elementos más representativos de cada investigador, para posteriormente tener claro en que consiste dicha temática que en este caso es el estudio de mercado, tema que se vincula directamente con la propuesta de la presente investigación. 
Tabla 2. Matriz conceptual del estudio de mercado.

\begin{tabular}{|c|c|c|c|c|}
\hline Autores & Vela (2012) & Espinoza (2013) & Flassler (2013) & $\begin{array}{c}\text { Salazar y Yánez } \\
(2014)\end{array}$ \\
\hline $\begin{array}{c}\text { Vela } \\
(2012)\end{array}$ & $\begin{array}{l}\text { Lugar en donde } \\
\text { asisten la oferta y la } \\
\text { demanda para realizar } \\
\text { la transacción de } \\
\text { bienes y servicios a un } \\
\text { determinado precio. }\end{array}$ & $\begin{array}{l}\text { Vela indica que es un } \\
\text { lugar donde están } \\
\text { presentes la demanda y } \\
\text { la oferta y Espinoza } \\
\text { menciona un } \\
\text { componente más como } \\
\text { es la competencia. }\end{array}$ & $\begin{array}{lr}\text { Ambos } & \text { conceptos } \\
\text { determinan } & \text { a los } \\
\text { bienes y } & \text { servicios } \\
\text { dentro del } & \text { mercado } \\
\text { turístico. } & \end{array}$ & $\begin{array}{l}\text { Son conceptos } \\
\text { con } \\
\text { componentes } \\
\text { diferentes. }\end{array}$ \\
\hline $\begin{array}{c}\text { Espinoza } \\
\text { (2013) }\end{array}$ & $\begin{array}{l}\text { El concepto de Vela es } \\
\text { muy abarcador y } \\
\text { presenta varios } \\
\text { componentes que } \\
\text { permiten su desarrollo } \\
\text { a diferencia de Salazar } \\
\text { y Yánez. }\end{array}$ & $\begin{array}{l}\text { Estudia la demanda, la } \\
\text { competencia y la oferta. }\end{array}$ & $\begin{array}{l}\text { En este aspecto se } \\
\text { determina que ambos } \\
\text { mencionan al espacio } \\
\text { o lugar como mercado } \\
\text { donde se ofrecen } \\
\text { bienes y servicios }\end{array}$ & $\begin{array}{l}\text { Un lugar donde } \\
\text { interactúan } \\
\text { compradores y } \\
\text { vendedores. }\end{array}$ \\
\hline $\begin{array}{c}\text { Flassler } \\
(2013)\end{array}$ & & $\begin{array}{l}\text { Palabra clave del } \\
\text { concepto determinación } \\
\text { del espacio. }\end{array}$ & $\begin{array}{l}\text { Determina el espacio } \\
\text { que ocupa un bien o } \\
\text { servicio en un mercado } \\
\text { específico. }\end{array}$ & $\begin{array}{lc}\text { Mencionan } & \text { tres } \\
\text { componentes } & \\
\text { claves del } & \text { del } \\
\text { estudio de } \\
\text { mercado. }\end{array}$ \\
\hline $\begin{array}{l}\text { Salazar y } \\
\text { Yánez } \\
\text { (2014) }\end{array}$ & & $\begin{array}{lr}\text { Ambos analizan la } \\
\text { demanda, la oferta y la } \\
\text { competencia como } \\
\text { componentes } \\
\text { integradores } \\
\text { mercado. }\end{array}$ & $\begin{array}{l}\text { La determinación de } \\
\text { los conceptos que } \\
\text { muestran ambos } \\
\text { autores se presenta de } \\
\text { forma más puntual. }\end{array}$ & $\begin{array}{ll}\text { Es el análisis de } \\
\text { la oferta, la } \\
\text { demanda y la } \\
\text { competencia. }\end{array}$ \\
\hline
\end{tabular}

Fuente: elaboración propia.

Al ser evaluada la matriz conceptual se identifican las semejanzas y diferencias que tienen los cuatro autores, la mayor y más notoria semejanza es que, Salazar y Yánez (2014), Fassler (2013), Vela (2012) y Espinoza (2013) es sobre el análisis de la matriz que consiste en estudio de mercado y la forma de analizar la oferta de un destino, determinando que tipo de bienes y servicios son demandados y cuál es la competencia que se encuentra en el lugar o espacio donde asisten los demandantes y hacen las transacciones y adquisición del bien o servicio; el estudio de mercado conlleva a determinar cuál es la interacción que tiene la demanda, la oferta y la competencia para el desarrollo de un destino turístico.

Una vez analizado los conceptos anteriormente expuestos se propone para el desarrollo de la investigación el siguiente diseño metodológico. 
Tabla 3. Desarrollo metodológico.

\begin{tabular}{|c|c|c|}
\hline FASES & ACTIVIDADES & $\begin{array}{l}\text { HERRAMIENTAS } \\
\text { INSTRUMENTOS }\end{array}$ \\
\hline $\begin{array}{l}\text { FASE I: Diagnóstico } \\
\text { situacional del entorno }\end{array}$ & $\begin{array}{l}\text { 1. Generalidades del cantón Chone } \\
\text { 2. Determinación de atractivos } \\
\text { turísticos. } \\
\text { 3. Características de los } \\
\text { alojamientos turísticos. }\end{array}$ & $\begin{array}{l}\text { Investigación de campo. } \\
\text { Revisión Bibliográfica. } \\
\text { Catastro turístico. }\end{array}$ \\
\hline $\begin{array}{l}\text { FASE II: Análisis de la oferta, } \\
\text { demanda y competencia }\end{array}$ & $\begin{array}{l}\text { 4. Determinar la oferta. } \\
\text { 5. Estudio de la demanda } \\
\text { 6. Estudio de la competencia. }\end{array}$ & $\begin{array}{l}\text { Catastro turístico del cantón } \\
\text { Chone, Tosagua y Bolívar. } \\
\text { Matriz Perfil Competitivo } \\
\text { Observación científica. } \\
\text { Encuestas }\end{array}$ \\
\hline
\end{tabular}

FASE III: Diseño del mix del marketing
7. Estudio y determinación del producto o Resort.

8. Posicionamiento del logo y slogan.

9. Estudio y posicionamiento del precio.

10. Distribución del producto.

11. Análisis y determinación de la promoción.

Fuente: elaboración propia.

Aplicación del mix de marketing.

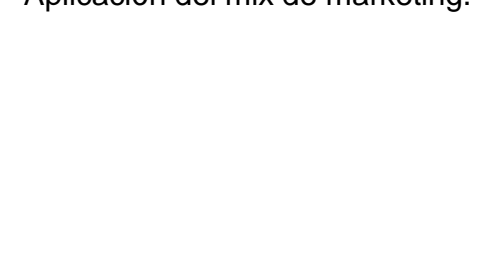

\section{RESULTADOS Y DISCUSIÓN}

Fase I: Diagnostico situacional del entorno.

La información obtenida dentro de esta fase muestra las generalidades y aspectos más relevantes del cantón Chone.

Ubicación.

- Provincia: Manabí

- Cantón: Chone

- Sitio: El Limón

- Latitud: : -0.7 (-0.7116897671068066,

- Longitud: -80.1 (-80.10639309883118)

El último censo del año 2010 determinó que el cantón Chone cuenta con una población de 126.5 mil hab. (9.2 \% respecto a la provincia de Manabí). La accesibilidad al cantón se encuentra principalmente enmarcada por la vía que conecta por el Suroeste con Portoviejo, Montecristi, Manta y la provincia del Guayas y por el Norte con Flavio Alfaro, El Carmen, Santo Domingo de los Tsáchilas y la provincia de Pichincha. 
Generalidades del cantón Chone.

Chone es el tercer cantón de Manabí en población y en economía, tiene una superficie de 3.570 kilómetros cuadrados. Cuenta con 7 parroquias rurales: Boyacá Convento, Canuto, Eloy Alfaro Ricaurte, San Antonio y Chibunga y dos urbanas: Chone y Santa Rita. Gobierno Municipal del Cantón Chone (2010).

\section{Sector productivo del cantón Chone.}

En la siguiente tabla se puede observar los cultivos producidos así como las hectáreas que se ocupan y las toneladas métricas promedio de producción. En el cantón se realizan otras actividades productivas o de servicio las cuales se exponen en el siguiente cuadro.

Tabla 4. Actividades productivas del cantón.

\begin{tabular}{|c|c|c|c|c|}
\hline Valor de la producción bruta & VPB-(millón) & $\%$ & PIB (millón) & $\%$ \\
\hline Agricultura, caza y pesca & 63,09 & 29,59 & 49,12 & 36,10 \\
\hline Minas y canteras & 0,03 & 0,01 & 0,04 & 0,02 \\
\hline Manufactura & 13,02 & 6,10 & 4,98 & 3,70 \\
\hline Electricidad, gas y agua & 2,30 & 1,10 & 0,77 & 0,60 \\
\hline Construcción & 11,02 & 5,15 & 5,12 & 3,76 \\
\hline $\begin{array}{c}\text { Comercio, bares y restaurantes } \\
\text { Transporte, almacenamiento y } \\
\text { comunicación }\end{array}$ & 46,09 & 21,50 & 24,18 & 17,77 \\
\hline Financiero & 27,63 & 13,00 & 17,82 & 13,10 \\
\hline servicios & 0,52 & 0,24 & 1,85 & 1,35 \\
\hline Total & 50,03 & 23,40 & 32,16 & 24,00 \\
\hline
\end{tabular}

Fuente: elaboración propia.

Al analizar la tabla anterior se puede identificar que el mayor rubro económico en el cantón Chone, lo componen las actividades de agricultura, caza, pesca y actividades turísticas, con un gran aporte económico en el Cantón, ocupando el comercio, bares y restaurantes el tercer lugar en la región.

Determinación de los atractivos turísticos.

El cantón Chone tiene un sin número de sitios turísticos para ser visitados los cuales no son muy conocidos; cabe destacar que la mayoría de los atractivos no cuenta con un inventario turístico actualizado, en los que se puede mencionar, el humedal "La Segua", Café color tierra, cabañas el Toto, cantiles el jobo, monumento a los raidistas, entre otros. 
Fase II: Análisis de la oferta, demanda y competencia.

Existe una gran afluencia de visitantes, a pesar de que el cantón Chone solo cuenta con una operadora turística que lo promociona, tiene un innumerable número de sitios turísticos para visitar entre los cuales se encuentran.

Tabla 5. Atractivos turísticos más conocidos del cantón Chone.

\begin{tabular}{|c|c|}
\hline Atractivos & Características \\
\hline Humedal "La Segua" & $\begin{array}{l}\text { El humedal La Segua brinda la facilidad de realizar paseos en canoa, } \\
\text { observación de aves, paisajes y degustación de chames. }\end{array}$ \\
\hline $\begin{array}{c}\text { Centro recreacional "Café } \\
\text { Color Tierra" }\end{array}$ & $\begin{array}{l}\text { Balneario natural a orillas del rio mosquito, en el interior de esta propiedad } \\
\text { se puede disfrutar de más de } 10 \text { cascadas y el ascenso de la montaña. } \\
\text { Comida criolla y artesanías. }\end{array}$ \\
\hline Cabañas "El Toto" & $\begin{array}{l}\text { Ofrece comidas típicas, balneario fluvial, áreas verdes, sitio de } \\
\text { esparcimiento. }\end{array}$ \\
\hline Los Raidistas & $\begin{array}{l}\text { En homenaje a Carlos Alberto Aray, Juan de Dios Zambrano, César Artemio } \\
\text { Aray, Emilio Hidalgo y Plutarco Moreira Barreiro, quienes demostraron que } \\
\text { era factible construir la carretera Chone - Quito. }\end{array}$ \\
\hline $\begin{array}{l}\text { Iglesia Santa Rosa de } \\
\text { Canuto }\end{array}$ & $\begin{array}{l}\text { De estilo predominante neo-clásico y construido con materiales de alta } \\
\text { calidad. }\end{array}$ \\
\hline
\end{tabular}

Fuente: elaboración propia.

Estudio de la demanda turística existente en el cantón Chone.

El estudio de la demanda es de gran importancia para las investigaciones turísticas, ya que ayudan a analizar el mercado. Gracias a este estudio se pudo determinar la aceptación de la inserción de una nueva propuesta la cual posee elementos tangibles e intangibles para la satisfacción de la demanda turística; según datos aportados en una entrevista realizada a la Directora del Ministerio de turismo de la zona 4, la cantidad de turistas que visitaron el cantón Chone en el año 2014, ascendió a 9859 personas, tomándose esta cifra como base de cálculo del tamaño de la muestra de población a encuestar para determinar la demanda.

Se realizó un total de 147 encuestas, las mismas que permitieron conocer que la edad promedio de los turistas que llegan al cantón Chone esta entre los 25 a 35 años ya que constituye el $45 \%$ del total. El tiempo de permanencia regular de los turistas es de 0 a 3 días, esto corresponde al $51 \%$ de los encuestados, la mayor motivación del viaje se da con fines de ocio en un 32\%. La aceptación que los turistas tienen en cuanto al Resort es del $83 \%$ los cuales afirman tener interés por hospedarse en un Resort, lo cual es importante para la propuesta. 
Estudio de la competencia en el alojamiento turístico.

En Chone al poseer sitios turísticos para ser visitado se debe considerar la idea de implementar un espacio de descanso para los visitantes, ya que solo cuenta con dos alojamientos catalogados como hoteles los cuales no están registrados en el catastro turístico 2016 y siete hostales que si están registrados y que posee una planta habitacional limitada.

Una vez analizada la competencia directa existente para el Resort se determina que en el cantón Chone hay establecimientos de hospedaje que no son óptimos puesto cuentan con servicios adecuados y lejos del estandar de un Resort, por esta razón se plantea que la competencia directa es débil lo que refuerza la propuesta de esta investigación.

Para analizar la competencia indirecta se aplicó la Matriz de perfil competitivo (MPC) de los cantones más cercanos a Chone, a continuación se muestran los pasos aplicados en la realización de la matriz.

Según Fred (2013) citado por Ganchozo (2015) es necesario identificar a los principales competidores, así como sus fortalezas y debilidades específicas en relación con la posición estratégica del lugar en estudio, los valores y los puntajes del total. En la evaluación se tomó en cuenta la siguiente clasificación en el puntaje 1 baja importancia, 2 media importancia, 3 considerable importancia, 4 alta importancia.

Según el análisis realizado de la matriz MPC, Chone tiene un resultado ponderado de 3,10 con ventajas al competidor número 1, que es el cantón Bolívar el cual tiene un resultado ponderado de 2,70 siendo la calidad de la producción su mayor fortaleza, con el competidor número 2 que es Tosagua que está en una posición desventajosa con 1,80 de ponderación. Uno de los ítem fuerte de Chone es el potencial de los recursos turísticos que se sitúa en un 0,60 a respecto de 0,40 de Bolívar y 0,20 de Tosagua, lo que contrasta en que los servicios turísticos de hospedaje es débil, con una ponderación de 0,20 al igual que Tosagua, con respecto a Bolívar sobresale con una ponderación de 0,30 , lo que refuerza la hipótesis de la creación de un hotel Resort en el cantón Chone.

Fase III: Diseño del mix del marketing.

Estudio y determinación del producto.

Objetivo: Lograr la completa satisfacción del cliente interno y externo, generando un buen servicio de calidad, brindando una instalación con los servicios requeridos para un hospedaje de este tipo.

Estrategias para el producto 
- Desarrollo de productos innovadores que involucren a la comunidad mejorando el servicio de alojamiento, puesto que se busca perfeccionar y crecer en el ámbito social y de esta manera incrementar los beneficios y la satisfacción del cliente.

Posicionamiento del logo y slogan.

El logo está conformado por un árbol en representación del área rural existente en el cantón Chone. El slogan, por su parte, expresa: "Tu comodidad en nuestras manos".

El slogan pretende generar confianza en los clientes y que conozcan que lo más importante de la empresa es la comodidad del turista.

\section{Estudio y posicionamiento del precio.}

Objetivo: Fijar precios similares a la competencia pero acorde a las instalaciones y al servicio de calidad que se brinda.

Estrategia:

- Adicionar tarifas a los servicios.

Plantear precios fijos a los servicios adicionales, como bebidas o platos típicos que no estén dentro del menú.

Distribución del producto o plaza.

Objetivo: Dar a conocer el producto a los clientes

Estrategias

- Diseñar un sistema para hacer las reservas

El sistema que se diseñe ayudara al cliente realizar las reservas de una forma más fácil, rápida, sencilla y segura, lo cual genera más confianza al turista al momento de hacer su reserva.

Análisis y determinación de la promoción.

Objetivo: Dar a conocer los servicios que ofrece el Resort.

Estrategias:

El Resort se dará a conocer mediante los siguientes canales de distribución:

- Trípticos: los mismos que serán entregados en ferias de turismo, ferias ganaderas $u$ de otro tipo, centros educativos, operadoras turísticas ubicadas cercas del cantón Chone y en otras provincias. 
- Página Web en la que se situé toda la información de forma electrónica capaz de contener texto, sonido, videos, programas, enlaces, imágenes, y muchas otras cosas del Resort.

\section{CONCLUSIONES}

La investigación permitió conocer el entorno actual del cantón Chone, a nivel agrícola y económico y turístico para así determinar los atractivos que estarían involucrados en la propuesta. A partir del criterio de cuatros autores se determinó el diseño metodológico el cual cuenta de tres fases., siendo estas el diagnóstico situacional del entorno, análisis de la oferta, demanda y competencia y el diseño del mix del marketing. Mediante el análisis de la competencia, se evaluó la competencia directa, identificando los establecimientos turísticos del cantón, y en el caso de la indirecta se realizó una matriz de perfil competitivo (MPC) en la cual se estudió los establecimientos de los cantones Bolívar y Tosagua ,demostrándose que el cantón Chone posee ventajas con respecto a los cantones aledaños con relación al potencial de los recursos turísticos. Con la aplicación de las herramientas del mix del marketing se pudo validar la construcción de un Resort, determinando los servicios que ofrecería el establecimiento de hospedaje planteado.

\section{REFERENCIAS BIBLIOGRÁFICAS}

Espinoza (2013). Diseño de una hostería ecológica en el cantón Pedernales, provincia de Manabí. (En línea). EC. Consultado, 14 de Ene, 2016. Formato PDF. Disponible en: http://www.dspace.uce.edu.ec/bitstream/25000/2045/1/T-UCE-0004-9.pdf

Fassler (2013). Diseño de productos turísticos del Ecuador para el mercado alemán. (En línea). EC, Consultado, 14 de Ene, 2016. Formato PDF. Disponible en: http://dspace.espoch.edu.ec/bitstream/123456789/2970/1/23T0375\%20FASLER \%20DANIELA.pdf

GAD (2010) Gobierno Autónomo Descentralizado de Chone. (En línea). EC. Consultado, 17 de DIC, 2016. Disponible en: http://www.chone.gob.ec/index.php?gc=39

Ganchozo, M. y Loor, A. (2015). Plan estratégico para el desarrollo del turismo en la comunidad piedra de plata, cantón Pichincha provincia Manabí. (En Línea). Ec. Manabí. Consultado, 26 de Oct. 2016. Formato PDF. Disponible en: 
http://repositorio.espam.edu.ec/bitstream/42000/148/1/Miriam\%20Ganchozo\%20\%20Alfredo\%20Loor.pdf

INEC (2010). Fascículo Provincial de Manabí.

Ministerio de Turismo. (2015). (En línea). Consultado 6 de Jun, 2016. Formato PDF. Disponible en: www.quito-turismo.gob.ec/.../43-registro-turistico?...reglamento-dealoja...

Salazar y Yánez (2014). Diseño de una propuesta de desarrollo turístico en el cantón Yaguachi para el fomento del turismo local. (En línea). EC. Consultado, 14 de Ene, 2016. Formato PDF. Disponible en: http://repositorio.ucsg.edu.ec/bitstream/3317/2315/1/T-UCSG-PRE-ESP-AETH183.pdf.

Vela (2012). Diseño de un producto turístico para la conservación y uso del patrimonio cultural del centro de turismo comunitario san Virgilio, Cantón Arajuno provincia de Pastaza. (En línea). EC. Consultado, 14 de Ene, 2016. Formato PDF. Disponible en:

http://dspace.espoch.edu.ec/bitstream/123456789/1839/1/Tesis\%20Diego\%20Ma uricio\%20Vela\%20Sampedro\%20-\%20EIE.pdf.

\section{Licencia Creative Commons}

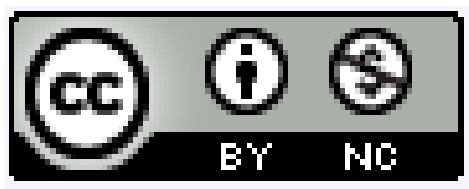

Revista Científica ECOCIENCIA está bajo una Licencia Creative Commons Atribución-NoComercial 4.0 Internacional. 